\title{
Partial trisomy and tetrasomy of chromosome 21 without Down Syndrome phenotype and short overview of genotype-phenotype correlation. A case report
}

\author{
Pavlina Capkovaa ${ }^{a}$ Nadezda Misovicova ${ }^{b}$, Dita Vrbicka ${ }^{a}$
}

\begin{abstract}
Aims. Trisomy of chromosome 21 is associated with Down syndrome (DS) - the commonest genetic cause of mental retardation. We report two unusual cases with partial trisomy of chromosome 21 and tetrasomy of chromosome 21 without DS phenotype. We include a short overview of the genotype-phenotype correlation studies in discussion.

Methods. Conventional chromosomal analysis, fluorescent in situ hybridisation (FISH), quantitative fluorescent PCR (QFPCR) and Nimblegen targeted chromosome 21 array were used for deciphering the genotypes.

Results. Conventional chromosomal analysis revealed one extra copy of derivative chromosome 21 in peripheral blood lymphocytes of the patients. FISH and QF PCR analyses identified duplicated loci (D21Z1, D21S1414, D21S1435) spanning from the centromere to band 21q21. Nimblegen targeted chromosome 21 array specified the range of duplication from the centromere to the band 21q21.3 (19 Mb) in the first case and the range of duplication and triplication resp from centromere to the bands $21 \mathrm{q} 21.3(15 \mathrm{Mb})$ and 21q11.2 (4 Mb) resp. in the second case. Additional material was of maternal origin in both cases. The different mechanisms led to the formation of the particular chromosomal imbalances. Conclusion. These findings confirm the conclusion of nonpresence of DS when bands 21q22.2 and 21q22.3 (Down critical region) are not duplicated. The patients had nonspecific phenotypes although some of their features such as "sandal gaps", joint hyperlaxity, hypotonia and brachycephaly are present in patients with DS. Our observation can help to narrow the region responsible for DS and to map the loci accountable for minor features of DS.
\end{abstract}

Key words: Down syndrome, partial trisomy/tetrasomy of chromosome 21, Down syndrome critical region (DSCR)

Received: May 9, 2013; Accepted: September 24, 2013; Available online: October 18, 2013

http://dx.doi.org/10.5507/bp.2013.077

${ }^{a}$ Department of Clinical Genetics and Fetal Medicine, University Hospital Olomouc and Faculty of Medicine and Dentistry, Palacky University Olomouc, Czech Republic

${ }^{b}$ Department of Clinical Genetics, University Hospital Martin, Martin, Slovak Republic

Corresponding author: Pavlina Capkova, e-mail:pcapkova@seznam.cz

\section{INTRODUCTION}

Chromosome 21 is the smallest human chromosome comprising about $1.4 \%$ of the total human genomic length. Trisomy 21 (Down Syndrome, DS, \#190685) is the major abnormality associated with this chromosome and is the commonest genetic cause of mental retardation, which affects 1.3 per 1000 livebirths $^{1}$. Although it is usually caused by the presence of an extra copy of chromosome 21, a subset of diagnostic features may be associated with the excessive presence of band 21q22 only. We report two cases with partial trisomy of chromosome 21 and tetrasomy of chromosome 21 without typical Down Syndrome (DS) phenotype.

\section{CLINICAL REPORTS}

\section{Case 1}

The patient - a 4 year old boy - was the first child of healthy nonconsanguineous parents. His mother was 25 years old at term. The pregnancy was uneventful; normal delivery was at $39^{\text {th }}$ week of gestation with a weight of $4150 \mathrm{~g}$, length $55 \mathrm{~cm}$ and head circumference (HC) of $35 \mathrm{~cm}$. Apgar score was 8, 9 and 10 . The baby required treatment for congenital stridor of larynx and pneumonia.
At the age of 4, the child had hypotonia, brachycephaly (head circumference $51 \mathrm{~cm}$ ), "sandal gaps" (gaps between toes 1 and 2), joint hyperlaxity and developmental delay with speech delay. Apart from brachycephaly no dysmorphic features were detected. Chromosomal analysis revealed an extra copy of chromosome 21 .

\section{Case 2}

The first child of a 31 year old mother was examined at the age of 14 months for hypotonia and slightly delayed developmental milestones for the first time. "Sandal gaps" and macroglossia were also observed. Hypotonia with joint hyperlaxity persisted up to age 6 . The mental development of the patient was normal at the time. The family history was unremarkable except for one spontaneous abortion. The parents were healthy and nonconsanguineous. Chromosomal analysis revealed an extra copy of chromosome 21.

\section{MATERIAL AND METHODS}

Chromosome analyses were performed on samples of the probands and their parents on peripheral blood lymphocytes using standard techniques. 
Fluorescence in situ hybridisation (FISH) studies were carried out with probes LSI 21q22.2 (MNB,D21S270, D21S337,D21S55,D21S233,GIRK2) (Cytocel1, Cambridge, UK), LSI 21q22.13-22.2 (D21S259,D21S341, D21S342) (Vysis, USA), CEP13/21(D13Z1/D21Z1) (Cytocell, Cambridge, UK) according to manufacturer's specifications. Chromosomes were counterstained with DAPI and hybridised metaphase spreads were analyzed using an epifluorescence microscope.

Genomic DNA was extracted from peripheral blood lymphocytes of the patients and both parents. Polymorphic markers from chromosome 21: D21S1414, D21S1435,D21S1411,D21S1446 (Sigma, Aldrich) and penta D (Promega) were studied by quantitative fluorescent PCR (QFPCR).

Niblegene targeted array was performed by the Department of Human Genetics University Medical Centre Nijmegen as the patients were involved in HSA21 research study AnEUploidy project.

\section{RESULTS}

\section{Cytogenetic resuts}

Case 1: the karyotype of the patient was $47, \mathrm{XY},+$ $\operatorname{der}(21)($ pter $\rightarrow q 21)$. Normal karyotype was found in the father whereas the mother was revealed to carry a balanced cryptic translocation of chromosome 21 and 15 . Thus her karyotype was 46,XX,t(15;21)(p11.2;q21).ish $\mathrm{t}(15 ; 21)(\mathrm{MNBx} 2$, GIRKx2)

Case 2: the karyotype of the patient showed the presence of an extra copy of chromosome $21: 47, \mathrm{XY},+$ psu $\operatorname{idic}(21)(q 21)$.

The karyotypes of the parents were 46,XX and 46,XY.

\section{FISH studies}

Case 1: FISH study with probe CEP 13/21 and LSI $21 \mathrm{q} 22.2$ in the proband confirmed the presence of the third copy of chromosome 21 and the absence of terminal parts of the extra chromosome. The case was concluded as a partial trisomy of chromosome 21 which arose due to $3: 1$ segregation with tertiary trisomy. Karyotype was: 47,XY,+der(21)t(15;21)(p11.2;q21).ish dup(21)

(D 13Z1/D21Z1+,D21S270-, MNB-,D21S337-, D21S55-,D21S233-,GIRK-)[25]mat.

Case 2: FISH study in the patient with probe CEP 13/21 and LSI 21q22.2 confirmed the result of cytogenetic analysis and narrowed the loci which were tetrasomic and disomic: 47,XY,+psu idic(21)(q21).ish idic 21(q21) (D13Z1/D21Z1++,D21S270-,MNB-,D21S337-,D21S55-, D21S233-,GIRK-)[130] dn. However, the status of chromosome bands q11.2 and q21 was not exactly determined.

\section{Molecular analysis}

Polymorphic markers from 5 loci (bands 21q21, 21q22.3) of the chromosome 21 were analysed. Results are summarized in Fig. 1.

\section{Results of oligonucleotide array}

Niblegene targeted array mapped the breakpoints more precisely in the cytobands $21 \mathrm{q} 21.3$ in the first case and 21q11.2 and 21q21.3 resp. in the second case (Fig. 1).

Case 1: Karyotype was specified: 47, XY,+der(21) $\mathrm{t}(15 ; 21)(\mathrm{p} 11.2$; 21$)$.ish $\operatorname{dup}(21)(\mathrm{D} 13 \mathrm{Z1/D} 21 \mathrm{Z1}$, D21S270-,MNB-,D21S337-,D21S55-,D21S233-,GIRK-). arr 21q11.2q21.3(13,200,000-30,923,550)x3. The size of the duplication is about $19 \mathrm{Mb}$ (including centromere) (Fig. 1).

Case 2: Karyotype was specified: 47,XY,+psu idic(21) (q21).ish idic(21)(q11)(D13Z1/D21Z1++,MNB-,GIRK-). arr 21q11.2q11.2(13,200,000-16,064,300)x4, 21q11.2q21.3 $(16,064,300-31,167,150) \times 3$. The size of the triplication is about $4 \mathrm{Mb}$ (including centromere) and of the duplication about $15 \mathrm{Mb}$.

\section{DISCUSSION AND CONCLUSION}

In both cases, partial trisomy and tetrasomy of chromosome 21 were detected by FISH, QFPCR and specified by oligonucleotide DNA array. DS phenotype was not present in either of the probands with the exception of some "minor abnormalities" (Fig. 1).

The lack of Down syndrome phenotype was caused by an absence of the partial trisomy of bands $21 \mathrm{q} 22.2$ and 21q22.3 - Down syndrome critical region (DSCR) (ref. ${ }^{2}$ ). The precise description of loci responsible for partial features of DS is a subject of many studies but genes responsible for them are still unknown. The analysis of several overlaping segmental trisomies 21 has led to suggestion that DS phenotype is associated with several dosage sensitive genes on chromosome 21 which, when present in three copies, cause the many different phenotypes seen in DS.

The cases of duplication of chromosome 21 excluding band 21q22 did not show the Down syndrome phenotype but instead, a rather unremarkable clinical pattern ${ }^{3,4}$. Their faces appeared normal or showed very mild and non-specific dysmorphic traits such as: hypertelorism, epicanthic folds, strabismus, mildly dysmorphic ears, short nose with depressed bridge etc. Most patients were mildly retarded with IQs between 50 and 70 (ref. ${ }^{5}$ ). Speech was especially delayed and defective, and in most patients particular behavioural abnormalities were observed, such as motor incoordination, restlessness and etc. ${ }^{3,6-8}$. Another patient with trip(21)(pter-q22.1) idic(21)(q22.1) did not exhibit Down syndrome phenotype; however brachycephaly, round face and prominent lower lip were described ${ }^{6}$. Duplication extending to band 21q22.1 shows strong association with severe mental retardation without dysmorphism consistent with DS (ref. ${ }^{9,10}$ ). Nineteen of typical Down features could be assigned to just 2 regions: short stature, joint hyperlaxity, hypotonia, major contribution to mental retardation and 9 anomalies of the face, hand and foot to the region D21S55, or DSCR, located on q22.2 or very proximal q22.3, and spanning 0,4-3 Mb; six facial and dermatoglyphic anomalies to the region D21S55-MX1, 


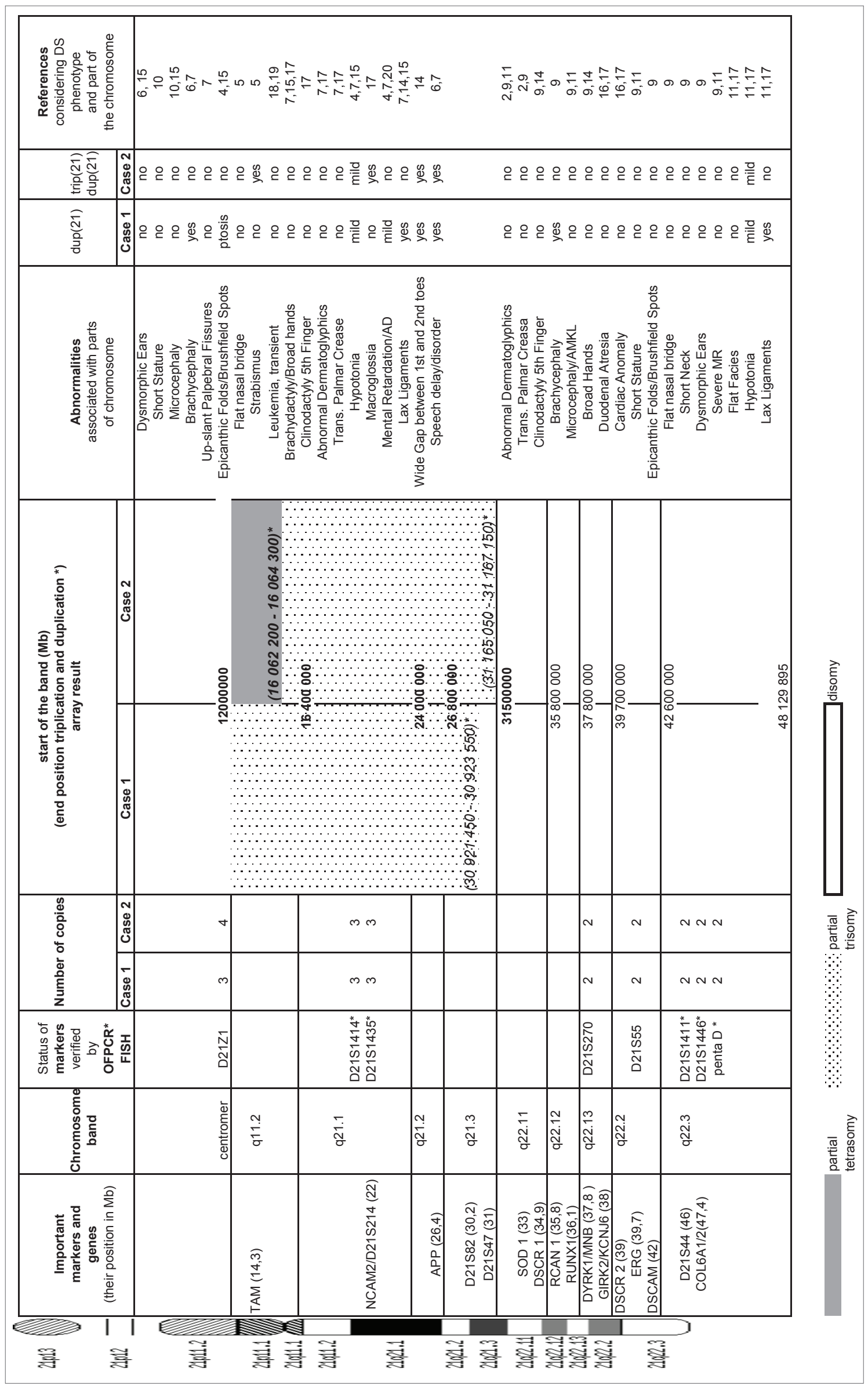

Fig. 1. Genotype - phenotype correlation in case 1 and case 2 . 
including the DSCR and spanning maximum of $6 \mathrm{Mb}$ on q22.2 and part of 22.3 (ref. ${ }^{11,12}$ ). We detected hypotonia, "sandal gap" and joint hyperlaxity in both probands but duplication D21S55 was not present (Fig. 1). Antanorakis described three patients with partial trisomy 21, which can extend as far distally as locus D21S82 without DS. The area between D21S82 and D21S55 was determined as the area of transition between two and three copies. The genetic distance between these loci is approximately $12 \mathrm{cM}$ and physical distance is estimated to be approximatelly $10 \mathrm{Mb}$ (ref. ${ }^{13}$ ). Minimal region of chromosome 21 which, when duplicated, generates the facial features, heart defect, mental retardation, and probably several of the dermatoglyphic changes of DS may include parts of bands 21q22.2 and 21q22.3, but it must exclude the genes SOD1(CuZn-superoxide dismutase mapping in 21q22.1) and APP (amyloid precursor protein mapping in 21q21.2) and most of the band 21q22.1, specifically the region defined by SOD1, SF57 and D21S47 ( ref. $^{2,14}$ ).

Our observation can definitely exclude the area involving genes APP and area involving loci D21S47 and D21S82 from DS phenotype. However, some facial features (brachycephaly, macroglossia, etc.) and further minor abnormalities (hypotonia, joint hyperlaxity) are determined by loci proximal to DSCR 1, which is consistent with our observation $7,14,15$.

In recent studies the nonexistence of single region (DSCR) responsible for all the most severe DS features has been expressed ${ }^{16,17}$. This is supported by fact that four of the major DS phenotypes: DS-specific congenital heart disease (DSCHD), Hirschsprung disease (HSCR), DS associated leukemia and Alzheimer's disease (AD) have never been associated with a particular region in chromosome 21. KCNJ6 (potassium inwardly-rectifying channel), RCAN1 (regulator of calcineurin), COL6A1/2 (collagen VI) and DYRK1A (dual- specifity tyrosine - regulated kinase 1A) were previously suggested to be critical for DSCHD. But DSCAM (DS cell adhesion molecule), as it is known to be highly expressed in the developing heart, is a likely candidate for $i^{16}$. The same gene is suggested as candidate gene for HSCR (ref. ${ }^{16}$ ). The genes RUNX1 (runt-related transcription factor), ERG (erythroblastosis virus E26) might be responsible for DS-associated leukemia but it excludes the necessary role of TIAM1(T cell lymphoma invasion and metastasis 1) and $\mathrm{BACH} 1$ in acute megakaryocytic leukemia (AMKL) (ref. ${ }^{16}$ ).

It was found that the mode of inheritance of centromeric chromosomal markers was compatible with duplication of one parental chromosome 21 in 9 DS patients with transient myeloproliferative syndrome. For these reasons, the hypothesis of 'disomic homozygosity' of a mutant gene on chromosome 21 as the causative mechanism was proposed in Down syndrome patients ${ }^{18}$. However neither of our patients suffered from transient abnormal myelopoiesis (TAM).

One possible explanation is that the cytogenetic abnormalities of our cases occured through meiosis I error. Cytogenetic and molecular studies demonstrated that in Down syndrome associated with TAM, trisomy 21 had arisen much more frequently through mitotic (or meiosis II) nondisjunctions than through meiosis I errors ${ }^{19}$.

The APP gene seems to be a candidate for AD. Nevertheless it is highly probably that more than one region exists ${ }^{16}$. While genetic studies have clearly confirmed the importance of triplication of the APP gene, in the pathogenesis of early onset AD in DS patients, the contribution of other genes is still largely undefined. It has been previously shown that increased dosage of two genes, RCAN1 and DYRK1, that are found on chromosome 21, cooperatively reduces the activity of the calcineurin/ NFAT-signaling pathway during embryonic development. The studies suggest that perturbation of the calcineurin/NFAT genetic circuit contributes to many of the developmental phenotypes observed in DS. Calcineurin, RCAN1 and DYRK1 have all also been implicated in $\mathrm{AD}$ pathogenesis ${ }^{20}$. However, the results regarding their molecular and cellular role in AD (AD associated with DS or sporadic AD) has neither been systematically studied in genetic loss- of-function (LOF) or gain-of-function (GOF) mouse models nor in trisomy 21 patient cells and CNS tissues. Only the APP gene was duplicated without DYRK1 and RCAN1 in both our patients, which could be explanation for the fact that neither of the patients suffered from early onset of AD.

Although there are many common features in our patients the mechanisms, which led to the formation of the abnormalities, are different. The additional derivative chromosome in the case 1 reflected a tertiary trisomy due to 3 : 1 meiosis I malsegregation. Meiotic nondisjunction is the cause of the presence of the extra copy of chromosome 21 in case 2 . As the orientation of the duplicated loci on derivative chromosome was not studied, only hypothetical explanation of the origin of duplication/ triplication can be proposed. The extra chromosome 21 was dicentric with interstitial supposedly inverted duplication and terminal deletion. Two mechanisms come into consideration. In one mechanism, either parent carries a paracentric inversion. This results in formation of a loop during meiotic pairing with a recombination event occuring in the loop. In the second mechanism, inverted low copy repeats in the same chromosome arm allow partial folding of one homologue onto itself with a recombination event between the inverted repeats. These two mechanisms require that a single copy region exists between the duplicated and deleted regions on the derivative chromosome $^{21}$. The presence single copy between duplicated and deleted part of the derivative chromosome was confirmed by array. However, the first mechanism can be excluded as neither of parents was a carrier of inversion.

\section{URLS}

Database of Genomic Variants

(http://projects.tcag.ca/variation/).

GeneLoc

(http://genecards.weizmann.ac.il/geneloc). 


\section{ACKNOWLEDGEMENTS}

We are grateful to the B. van Bon from Department of Human Genetics University Medical Centre Nijmegen (NL) for array results.

\section{CONFLICT OF INTEREST STATEMENT}

The authors state that there are no conflicts of interest regarding the publication of this article.

\section{REFERENCES}

1. Delacruz FF, Gerald PS. Down Syndrome; Research perspectives. Baltimore: University Park Press;1981.

2. Korenberg JR, Kawashima H, Pulst SM. Molecular definition of a region of chromosome 21 that causes features of the Down syndrome phenotype. Am J Hum Genet 1990;47:236-46.

3. Chen $\mathrm{H}$, Tyrkus M, Woolley PV. Double aneuploidy: partial trisomy 21 and XO/XXX in a family with 12/21 translokation. Ann Genet 1978:21:177-80.

4. Rauch A, Pfeiffer RA, Trautmann U, Liehr T, Rott HD, Ulmer R. A study of ten small supernumerary (marker) chromosomes identified by fluorescence in situ hybridization. Clin Genet 1992,42:84-90.

5. Schinzel A. Catalogue of unbalanced chromosomal aberrations in man. $2^{\text {nd }}$ Revised and Expanded Edition New York: Walter de Gruyter; 2001.

6. Cerretini R, Luccerini V, Stivel M. Tetrasomy 21 pter-->q22.11, Molecular, cytogenetics and clinical findings. Clin Genet 1999;55:283-6.

7. Daumer-Haas C, Schuffenhauer S, Walter JU, Schiller RD, Porstmann T, Korenberg JR. Tetrasomy 21 pter-->q22.1 and Down syndrome: molecular definition of the region. Am J Med Genet 1994;53:359-65.

8. Hagemeijer A, Smit EME. Partial trisomy 21. Futher evidence that trisomy of band 21q22 is essential for Down's phenotype. Hum Genet 1977;38:15-23.

9. Nadal M, Moreno S, Pritchard M, Preciado MA, Estivill X, RamosArroyo MA. Down syndrome: characterisation of a case with partial trisomy of chromosome 21 owing to a paternal balanced translocation $(15 ; 21)$ (q26;q22.1) by FISH. J Med Genet 1997;34:50-4

10. Park JP, Wurster-Hill DH, Andrews PA, Cooley WC, Graham JM, Jr. Free proximal trisomy 21 without the Down syndrome. Clin Genet $1987 ; 32: 342-8$
11. Delabar JM, Theophile D, Rahmani Z. Molecular mapping of twentyfour features of Down syndrome on chromosome 21. Eur J Hum Genet 1993;1:114-24.

12. McCormic MK, Schinzel A, Petersen MB, Stetten G, Driscoll DJ, Cantu ES, Tranebjaerg L, Mikkelsen M, Watkins PC, Antonarakis SE. Molecular genetic approach to the characterization of the "Down syndrome region" of chromosome 21. Genomics 1989;5:325-31.

13. Antonarakis SE, Warren AC, McCormick M, Lewis JG, Hieter PA, Chakravarti A. Molecular and Cytogenetic studies of Non Disjunction. Alan R. Liss, Inc.;1989:29-43.

14. Korenberg JR, Bradley C, Disteche CM. Down syndrome: molecular mapping of the congenital heart disease and duodenal stenosis. Am J Hum Genet 1992;50:294-302.

15. Gutiérrez-Angulo M, Ramos AL, Dávalo N, Sánchez-Corona J, Rivera $H$. An extra idic $(21)(q 22,1)$ in child with some features os Down's syndrome. Clin Genet 1999;55:203-6.

16. Korbel JO, Tirosh-Wagner T, Urban AE, Chen XN, Kasowski M, Dai L, Grubert F, Erdman Ch, Gao MC, Lange K, Sobel EM, Barlow GM, Aylsworth AS, Carpenter NJ, Clark RD, Cohen MY, Doran E, FalikZaccai T, Lewin SO, Lott IT, McGillivray BC, Moeschler JB, Pettenati MJ, Pueschel SM, Rao KW, Shaffer LG, Shohat M, Van Riper AJ, Warburton D, Weissman S, Gerstein MB, Snyder M, Korenberg JR. The genetic architecture of Down syndrome phenotypes revealed by high-resolution analysis of human segmental trisomies. PNAS 2009;106:12031-6.

17. Korenberg JR, Chen XN, Schipper R, Sun Z, Gonsky R, Gerwehr S, Carpenter N, Daumer C, Dignan P, Disteche C. Down syndrome phenotypes: the consequences of chromosomal imbalance. Proc Natl Acad Sci USA 1994;91:4997-5001.

18. Niikawa N, Deng HX, Abe K, Harada N, Okada T, Tsuchiya H, Akabosh I, Matsuda I, Fukushima Y, Kaneko Y, Kuwano A, Kajii T. Possible mapping of the gene for transient myeloproliferative syndrome at 21q11.2. Hum Genet 1991,87:561-6.

19. Shen JJ, Williams BJ, Zipursky A, Doyle J, Sherman SL, Jacobs PA, Shugar A L, Soukup SW, Hassold TJ. Cytogenetic and molecular studies of Down syndrome individuals with leukemia. Am J Hum Genet 1995,56:915-25.

20. Kimura R, Kamino K, Yamamoto M, Nuripa A, Kida T, Kazui H, Hashimoto R, Tanaka T, Kudo T, Yamagata H, Tabara Y, Miki T, Akatsu H, Kosaka K, Funakoshi E, Nishitomi K, Sakaguchi G, Kato A, Hattori $\mathrm{H}$, Uema T, Takeda M. DYRK1A gene, encoded in chromosome 21 Down syndrome critical region, bridges between \{beta\}-amyloid production and tau phosphorylation in Alzheimer disease. Hum Mol Genet 2007;16:15-23.

21. Rowe $L R$, Lee JY, Rector $L$, Kaminsky EB, Brothman $A R$, Martin $C L$, South ST. U-type exchange is the most frequent mechanism for inverted duplication with terminal deletion rearrangements. J Med Genet 2009;46:694-702. 